Presented at: Annual Meeting, Computational Neuroscience

Brugge, Belgium, July 2000 .

\title{
Modeling Temporal Response Characteristics of V1 Neurons with a Dynamic Normalization Model
}

\author{
Samuel Mikaelian* and Eero P. Simoncelli \\ Center for Neural Science \\ New York University \\ 4 Washington Place \\ New York, NY 10003 \\ * Current Address: \\ Riverside Research Institute \\ 156 William St. \\ New York, NY 10038-2609
}

Abbreviated title: Model of Temporal Response Characteristics of V1 Cells

Keywords: V1, normalization, feedback, transients, dynamics 


\begin{abstract}
We present a dynamic normalization model to represent both the transient and the steady state components of V1 simple and complex cell responses. Primary receptive field properties are chiefly determined by the convergence of LGN afferents. These linear responses are rectified, and subjected to shunting inhibition through cortical feedback, which accounts for the non-linear characteristics of the neuronal responses. The duration of the transient response is determined by the time delay and the low-pass filtering of the cortical feedback. In addition to accounting for basic nonlinear behaviors such as response saturation and cross-orientation inhibition, the model is also able to reproduce several short-term contrast and pattern-selective adaptation effects.
\end{abstract}




\section{Introduction}

Normalization models have been used to account for various non-linear aspects of the steadystate responses of V1 simple and complex cells. In the simplest form of such a model, neural responses are computed by rectifying the responses of a set of oriented linear filters, and dividing each of these responses by the sum of the full set of responses [4]. Such a model can account for non-linear phenomena such as response saturation, cross-orientation suppression and contrast-dependent phase advance of simple cell responses. More recently, a patternselective normalization model, in which the divisive signal is a weighted sum of nearby neural responses, has been used to account for surround suppression [7]. None of these models, however, account for the dynamic temporal aspects of neural responses, such as transient responses after the onset or offset of stimuli, and short-term contrast and patternselective adaptation. If such effects are not within the scope of a functional description, it cannot meaningfully be connected with a more biophysically-detailed one. An earlier attempt at incorporating realistic temporal dynamics, successfully reproduced the typical transient response of an MT cell to a simple stimulus [5]. Here we propose a generalized normalization model that can capture a host of dynamical effects associated with V1 cells.

\section{The model}

The membrane potential is modeled through an RC-type circuit,

$$
C \frac{d V_{i}(t)}{d t}+g_{i}(t) V_{i}(t)=I_{i}(t)
$$

whose driving current is provided by the firing of LGN afferents [1,2]. The membrane conductance $g_{i}(t)$ is modulated by intra-cortical interactions, and is chiefly responsible for gain-control effects associated with normalization. The constant membrane capacitance $C$, introduced in Ref. [1] to account for the observed contrast-dependent phase advance of simple 
cell responses, is retained here. The neuron's instantaneous firing rate, $R_{i}(t)$, is obtained by half-wave rectification and squaring of the potential. For the case of modeling simple cell responses, the current $I_{i}(t)$ is represented by a linear sum of local stimulus contrasts filtered through a kernel that is the product of a spatial Gabor function and a causal temporal kernel with realistic parameter values. To obtain model complex cell responses, a quadrature pair of such filters is employed so as to render the input, $I_{i}(t)$, nearly independent of the overall spatio-temporal phase, à la energy models.

The various dynamical effects described here are attributable to the following functional dependence of the conductance,

$$
g_{i}^{2}(t)=\left\{\overline{g^{2}}(t-\Delta t) \sum_{j} w_{i j} R_{j}(t-\Delta t)\right\}_{l . p .}+\sigma^{2}
$$

where $\overline{g^{2}}$ indicates a uniform averaging of $g^{2}$ over the pool of neurons, $\Delta t$ is the time-delay in cortical feedback, l.p. denotes low-pass filtering, and $\sigma^{2}$ is the semi-saturation constant. The set of weights $w_{i j}$ allow for pattern-selectivity of the normalization. If the model response represents that of a simple or complex cell, the corresponding pool consists entirely of model simple or complex cells, respectively. Since in the case of modeling simple cells, for each neuron its quadrature companion is weighted equally in the pool, the conductance, unlike the response, is independent of spatio-temporal phase. (Note that the phase independence can also be simulated by an averaging over phases or spatio-temporal locations.) For simplicity, the frequency tuning of the neurons in the pool are fixed in our simulations, but the orientation tunings are allowed to cover the entire range, and the weights follow a normal distribution given by,

$$
w_{i j}=\exp \left(-\frac{2\left(\theta_{i}-\theta_{j}\right)^{2}}{\pi^{2}}\right)
$$

where $\theta$ denotes the preferred orientation (in radians). If the weights $w_{i j}$ are taken to be uniform, the model reduces to a standard normalization model when in steady state, thus, encompassing the effects previously studied via normalization. 


\section{Results}

Simulations of the model have so far been consistent with physiological data. Fig. 1 shows the response histogram of a directionally non-selective model complex cell to a stationary grating of optimal frequency and orientation lasting for $1.25 \mathrm{sec}$. It clearly shows a transient response followed by a sustained one. The duration of the transient is controlled by the time delay in cortical feedback, $0.05 \mathrm{sec}$ in this case, and the time constant of the low-pass filtering, $0.1 \mathrm{sec}$. The response during the steady state is noticeably reduced compared to the peak of the transient response, because cortical feedback has instituted the normalization and, consequently, gain control. Transient characteristics can be explored further. Because normalization has not yet taken effect, the early stage of the transient response shows no sign of contrast saturation. Even so, the orientation tuning curve based on the average firing rate obtained from the first $0.5 \mathrm{sec}$ of the response of the neuron of Fig. 1 is essentially identical in shape to that obtained from the steady-state portion of the response. This is evident from the normalized tuning curves in Fig. 2, supporting the inference made by Müller that orientation discrimination is as reliable during the transient stage as subsequently.

One can also examine response characteristics when combinations of gratings are presented either simultaneously or sequentially in time. Cross-orientation suppression refers to the suppression of the response to a stimulus moving in the preferred direction of the neuron if that stimulus is paired with one moving in an orthogonal direction. It has been shown previously that normalization provides a quantitative description of that effect during steady state. Correspondingly, in our model, this suppression is not displayed at the early stage of the transient response, when normalization is not yet operational. However, once normalization manifests itself during the transition from a transient response to the steady state, crossorientation suppression becomes evident.

With the stimuli presented sequentially in time, adaptation and "forward masking" effects 
may be explored. For example, Fig. 3 shows responses to a stationary grating with optimal parameters presented for $0.5 \mathrm{sec}$, followed by two similar probe gratings of $0.15 \mathrm{sec}$ duration presented $0.2 \mathrm{sec}$ and $2 \mathrm{sec}$ after the adapting grating. The diminished response to the first probe shows rapid contrast adaptation, a gain-control effect. That this effect should fade by the time the second probe is presented, is determined by our choice values for the timedelay and low-pass filtering constants. These parameters dictate which part of the neuron's histogram is contributing to the normalization term. The figure is consistent with the data in Ref. [6].

Our model can also reproduce the pattern-specific adaptation reported in Ref. [6]. Fig. 4 shows the orientation-tuning curves of a complex cell in the model, before (continuous curve) and after (dashed curve) adaptation to a stationary grating. The grating, lasting for 0.5 seconds, has its orientation offset from the optimal one for the cell by 14 degrees. Beyond the overall diminishing of the response, there is the relative suppression in the neighborhood of the adapting orientation. The model predicts that this "rapid adaptation" effect will not be evident during the interval (roughly $0.02 \mathrm{sec}$ ) immediately following the onset of the probe stimulus. Furthermore, this effect is present even if the weights $w_{i j}$ in Eq. 2 were chosen to be uniform. It arises due to the underlying dynamics of the normalization model. This is in contrast to recent accounts of such stimulus-specific adaptation in which the feedback (normalization) weights are adjusted according to image statistics [8] or short-term synaptic depression [3].

\section{Summary}

We have generalized the basic normalization model in a rather simple manner to account for the time course of V1 neuronal responses. Our model extends the range of observed non-linear behaviors encompassed by such a functional description and advances a step toward a more biophysical account of these mechanisms. But we have not yet determined a biophysically- 
detailed explanation, and the particular choice in Eq. 2 is somewhat ad-hoc. However, it can be shown mathematically that extending normalization models to incorporate patternselectivity is fairly constrained based on the steady-state responses they generate. It appears, then, that incorporating both biophysical and mathematical constraints should narrow the field of candidate models considerably. Our model can be further tested in a variety of ways, such as to what extent the myriad of effects observed in steady state carry over to the transient portion of the response. 


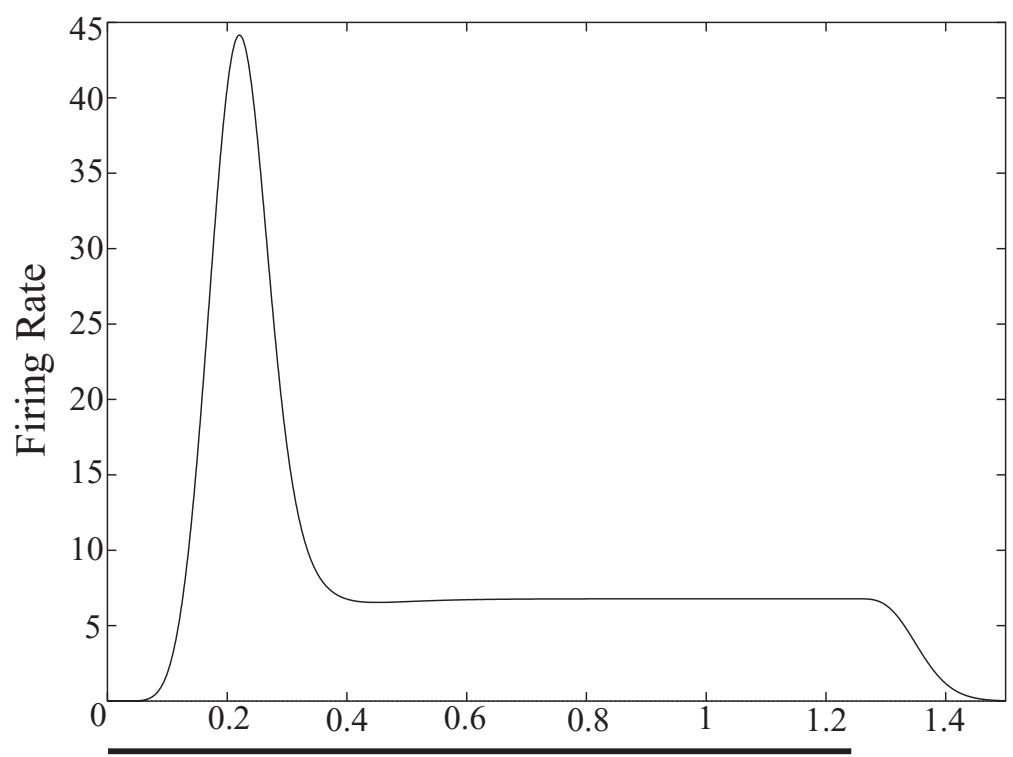

Time (sec)

Figure 1: Response histogram of a directionally non-selective model complex cell to a stationary grating of optimal frequency and orientation lasting for $1.25 \mathrm{sec}$. The time delay for cortical feedback is $0.05 \mathrm{sec}$ and the time constant of low-pass filtering is $0.1 \mathrm{sec}$. The time course of the stimulus is traced (heavy line) below the graph.

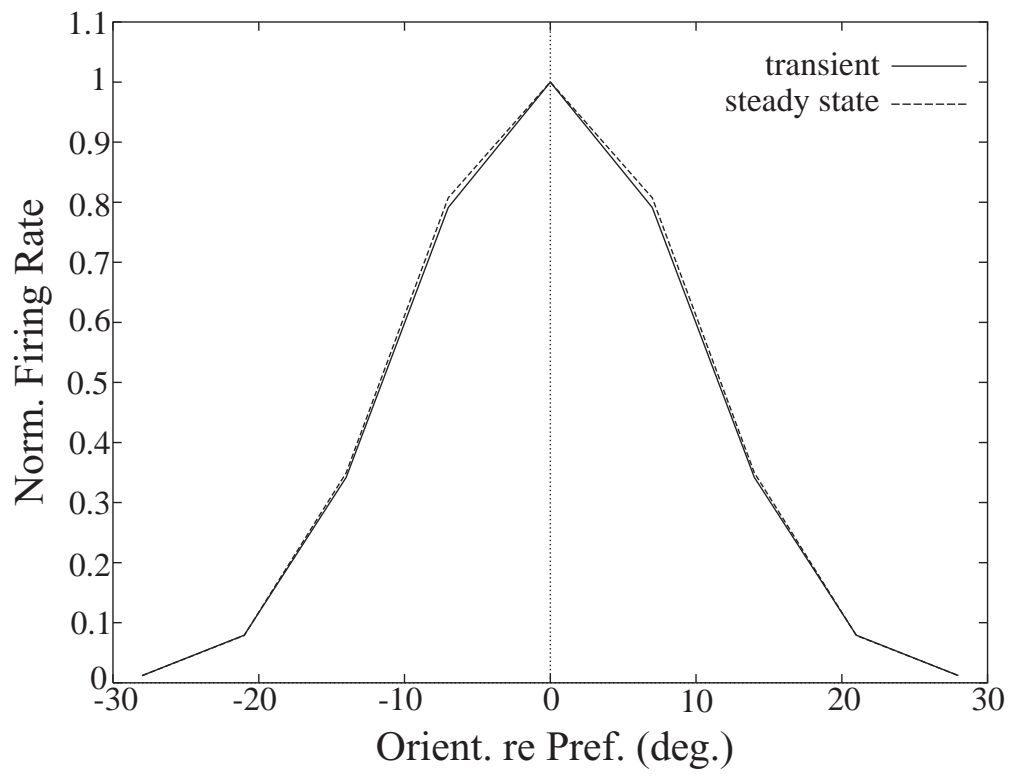

Figure 2: Normalized orientation tuning curves of the neuron of Fig. 1. The continuous and dashed curves correspond to the average response during the first $0.5 \mathrm{sec}$ and the subsequent portion of the histogram, respectively. The curves have been normalized such that their peak values are at one. 


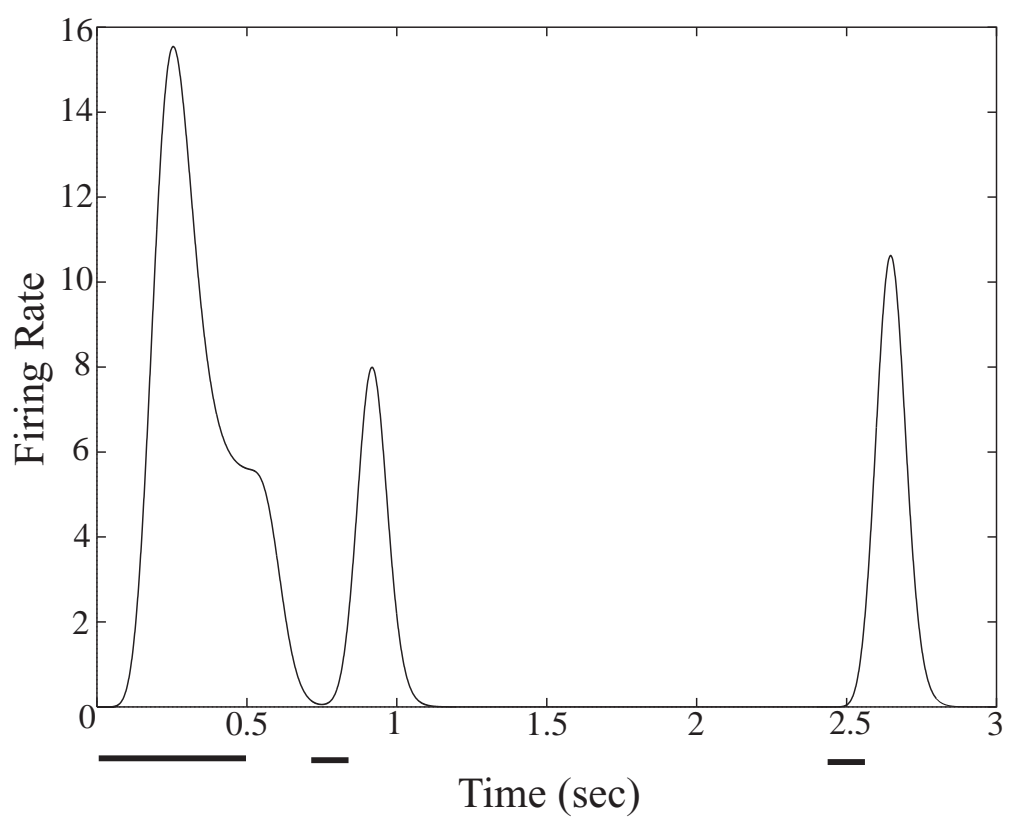

Figure 3: Response histogram of the neuron described in Fig. 1 to a stationary adapting grating presented for $0.5 \mathrm{sec}$, followed by two probe gratings $0.2 \mathrm{sec}$ and $2.0 \mathrm{sec}$ later, respectively, lasting for $0.15 \mathrm{sec}$ each. All stimuli have optimal frequency and orientation. The time course of the stimulus is traced (heavy line) below the graph.

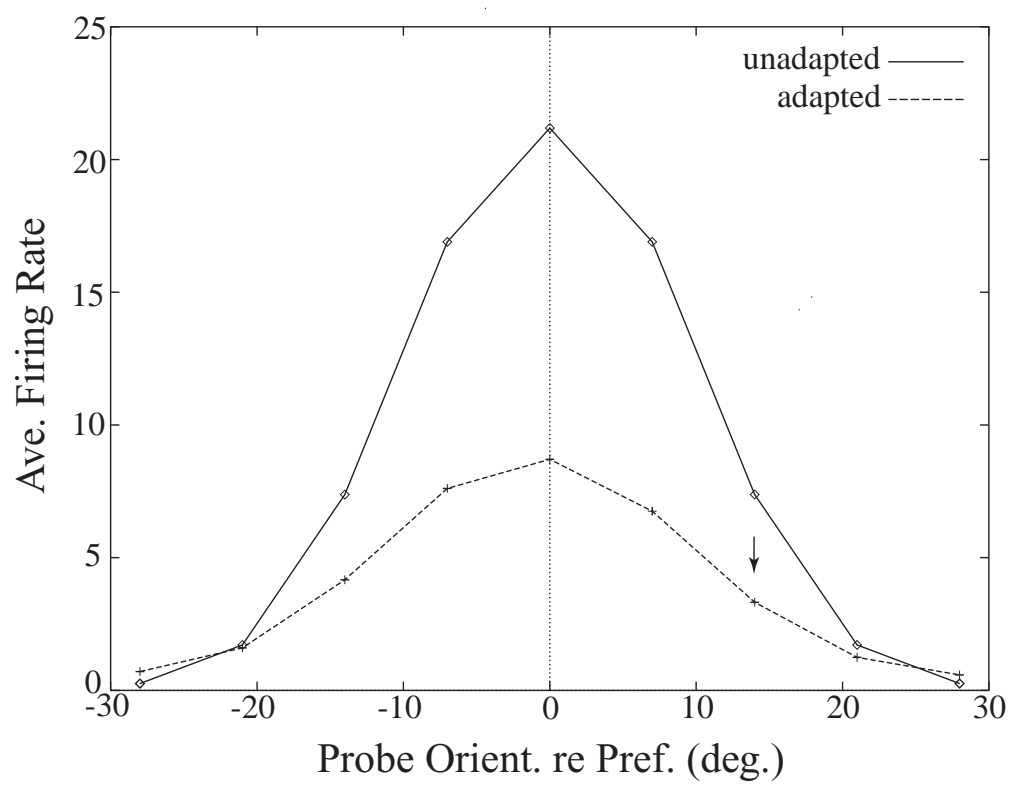

Figure 4: Orientation tuning curves of the neuron described in Fig. 1 before (continuous curve) and $0.2 \mathrm{sec}$ after (dashed curve) rapid adaptation to a stationary grating. The orientation of the adapting stimulus, which lasts for $0.5 \mathrm{sec}$ is indicated by the arrow. 


\section{References}

[1] M. Carandini and D. J. Heeger. Summation and division by neurons in primate visual cortex. Science, 264:1333-1336, 1994.

[2] M. Carandini, D. J. Heeger, and J. A. Movshon. Linearity and normalization in simple cells of the macaque primary visual cortex. Journal of Neuroscience, 17:8621-8644, 1997.

[3] F. S. Chance, S. B. Nelson, and L. F. Abbott. Temporal characteristics of V1 cells arising from synaptic depression. Computational Neuroscience, Trends in Research, pages 143-148, 1998.

[4] D. J. Heeger. Normalization of cell responses in cat striate cortex. Visual Neuroscience, 9:181198, 1992.

[5] S. Mikaelian, V. P. Ferrera, and E. P. Simoncelli. Modeling MT neuronal responses to compound stimuli. Society for Neuroscience Abstracts, 24:2095, 1998.

[6] J. R. Muller, A. B. Metha, J. Krauskopf, and P. Lennie. Rapid adaptation in visual cortex to the structure of images. Science, 285:1405-1408, 1999.

[7] E. P. Simoncelli and O. Schwartz. Image statistics and cortical normalization models. In M. S. Kearns, S. A. Solla, and D. A. Cohn, editors, Adv. Neural Information Processing Systems, volume 11, pages 153-159, Cambridge, MA, 1999. MIT Press. Presented at Neural Information Processing Systems, 1-3 Dec 1998.

[8] M. J. Wainwright and E. P. Simoncelli. Explaining adaptation in V1 neurons with a statistically optimized normalization model. In Investigative Opthalmology and Visual Science Supplement (ARVO), volume 40, pages S-573, May 1999. 
Samuel Mikaelian obtained his BA in physics and mathematics from U.C. Berkeley. He received a Ph.D. in physics from U. Florida in 1996. Subsequently, he served as a postdoctoral fellow at the Center for Neurobiology and Behavior at Columbia University and later at the Center for Neural Science at NYU. He is currently on the research staff at the Riverside Research Institute.

Eero Simoncelli started his higher education as a physics major at Harvard, went to Cambridge University on a Knox Fellowship to study Mathematics for a year and a half, and then returned to the States to pursue a doctorate in Electrical Engineering and Computer Science at MIT. He received his Ph.D. in 1993, and joined the faculty of the Computer and Information Science department at U. Pennsylvania. He came to NYU in September of 1996, as part of the Sloan Center for Theoretical Visual Neuroscience. He received an NSF Faculty Early Career Development (CAREER) grant in 1996, a Sloan Research Fellowship in 1998, and became a Howard Hughes Medical Institute Investigator in 2000. 